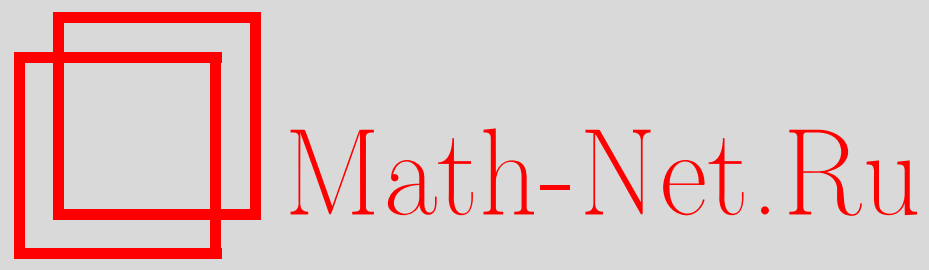

О. И. Мохов, О группах когомологий комплексов однородных форм на пространствах петель гладких многообразий, Функи. анализ и его прил., 1998, том 32, выпуск 3, 22-34

DOI: https://doi.org/10.4213/faa420

Использование Общероссийского математического портала MathNet.Ru подразумевает, что вы прочитали и согласны с пользовательским соглашением http://www . mathnet.ru/rus/agreement

Параметры загрузки:

IP : 35.173 .219 .149

26 апреля 2023 г., 17:33:51

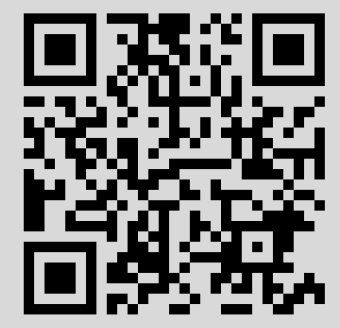




\title{
О группах когомологий комплексов однородных фором на пространствах петель гладких многообразий
}

\author{
(с) 1998. О. И. Мохов
}

Комплексы $\left(\Omega_{[m]}, d\right)$ однородных форм произвольного фиксированного порядка $m$ на пространствах петель гладких многообразий были введены в заметке автора [1], где анонсированы также некоторые результаты об их группах когомологий. Данная статья посвящена доказательству этих результатов и дальнейшему развитию теории однородных форм на пространствах петель гладких многообразий. Предлагаемый в статье подход к изучению топологии пространств петель тесно связан с интенсивными исследованиями последних лет различных однородных структур, возникающих в математической физике и теории поля. Начало этим исследованиям было положено в 1983 г. в статье Б. А. Дубровина и С. П. Новикова [2], где были введены и изучены невырожденные локальные однородные скобки Пуассона первого порядка

$$
\left\{u^{i}(x), u^{j}(y)\right\}=g^{i j}(u(x)) \delta_{x}(x-y)+b_{k}^{i j}(u(x)) u_{x}^{k} \delta(x-y), \quad \operatorname{det} g^{i j}(u) \neq 0,
$$

играющие важную роль в гамильтоновой теории систем гидродинамического типа, в частности, уравнений усреднения Уизема, описывающих эволюцию медленно промодулированных многофазных решений систем нелинейных уравнений [3-5]. В [2] Дубровин и Новиков сформулировали принцип сохранения гамильтоновости при усреднении системы, а именно, при усреднении гамильтоновых нелинейных систем, обладающих локальными гамильтоновыми структурами, они предложили процедуру усреднения соответствующих локальных скобок Пуассона. Эта процедура Дубровина-Новикова всегда приводит к однородным скобкам первого порядка, т.е. вида (0.1) (как доказал А. Я. Мальцев [6, 7], скобка, получаемая процедурой ДубровинаНовикова, действительно является скобкой Пуассона). В [2] доказано, что формула (0.1) задает скобку Пуассона, т.е. скобка (0.1) кососимметрична и удовлетворяет тождеству Якоби, тогда и только тогда, когда, во-первых, $g^{i j}(u)$ - произвольная плоская псевдориманова метрика (метрика нулевой римановой кривизны) и, во-вторых, $b_{k}^{i j}(u)=-g^{i s}(u) \Gamma_{s k}^{j}(u)$, где $\Gamma_{s k}^{j}(u)-$ риманова связность, порождаемая метрикой $g^{i j}(u)$ (связность Леви-Чивиты). Таким образом, любая скобка Пуассона вида (0.1) является постоянной в плоских координатах метрики $g^{i j}(u)$. Этот гамильтонов подход привел к

* Работа выполнена при частичной финансовой поддержке Российского фонда фундаментальных исследований, грант № 96-01-01623, INTAS, грант № 96-0770, и РФФИ-ННИО, грант № 96-01-00050G. 
построению С. П. Царевым теории интегрируемых диагонализуемых систем гидродинамического типа [5]. В качестве естественного обобщения локальных однородных скобок Дубровина-Новикова в работах автора и Е. В. Ферапонтова [8] (см. также [9-11]) были найдены и изучены нелокальные однородные скобки Пуассона, определенные на пространствах петель многообразий постоянной римановой кривизны (эти пуассоновы структуры также играют важную роль в теории систем гидродинамического типа и уравнений усреднения Уизема и допускают дальнейшие естественные однородные обобщения, изученные в [10]). Общие локальные однородные скобки Пуассона произвольных порядков были введены Дубровиным и Новиковым в [12], где была поставлена задача их классификации, которая на данный момент далека от своего полного решения. Полностью классифицированы только однородные скобки Пуассона нулевого (Дарбу), первого (Дубровиным и Новиковым [2]) и второго (Потеминым [13, 14], Дойлем [15]) порядков. Для однородных скобок Пуассона третьего порядка получены только частичные классификационные результаты (см. $[14,15])$. Однородные пуассоновы структуры третьего порядка играют важную роль в теории уравнений МонжаАмпера (см. [16]) и в теории уравнений ассоциативности в двумерной топологической теории поля [17]. Многомерные однородные скобки Пуассона изучены Дубровиным, Новиковым и автором $[12,18,21,22]$. Однородные симплектические структуры (замкнутые однородные 2-формы) произвольных порядков на пространствах петель гладких многообразий были введены и изучены автором в [19-24] в связи с исследованием гамильтонова формализма нелинейных сигма-моделей теории поля, определяющих, в частности, классическое действие бозонных струн, и их обобщений. Как показано автором в [19] (см. также [21-23]), гамильтонов формализм двумерных нелинейных сигма-моделей с кручением задается однородными симплектическими структурами первого порядка на пространствах петель псевдоримановых многообразий. В [19-22] полностью решена задача описания однородных симплектических (предсимплектических) форм первого и второго порядков на пространствах петель гладких многообразий (симплектические формы нулевого порядка классифицируются классической теоремой Дарбу конечномерной симплектической геометрии). Оказалось, что такие симплектические структуры порождают интересные дифференциально-геометрические объекты на многообразии и описываются инвариантным образом в терминах аффинных связностей со специальными свойствами на этом многообразии, ковариантных производных и тензоров кривизны и кручения связностей. В частности, симплектические структуры первого порядка классифицируются аффинными связностями на псевдоримановом многообразии, согласованными с метрикой и обладающими тензором кручения, определяющим замкнутую 3-форму на этом псевдоримановом многообразии $[19,21,22]$, а симплектические структуры второго порядка порождаются симплектическими связностями на почти симплектических многообразиях [20-22]. В данной статье введены произвольные однородные формы на пространствах петель гладких многообразий, построены комплексы однородных форм и в ряде случаев найдены их группы когомологий. 


\section{§1. Однородные формы на пространствах петель гладких многообразий}

Пусть $M$ - гладкое $N$-мерное многообразие с локальными координатами $u^{1}, \ldots, u^{N}$. Под пространством петель $\Omega M$ многообразия $M$ мы всегда будем понимать пространство всех гладких параметризованных отображений $\gamma$ окружности $S^{1}$ в $M, \gamma: S^{1} \rightarrow M, \gamma(x)=\left\{u^{i}(x)\right\}, x \in S^{1}$. Касательное пространство $T_{\gamma} \Omega M$ пространства петель $\Omega M$ в точке $\gamma$ состоит из всех гладких векторных полей $\xi=\left\{\xi^{i}, 1 \leqslant i \leqslant N\right\}$, определенных вдоль петли $\gamma$, причем $\xi(\gamma(x)) \in T_{\gamma(x)} M$ для любой точки $x \in S^{1}$, где $T_{\gamma(x)} M-$ касательное пространство многообразия $M$ в точке $\gamma(x)$.

ОПРЕДЕЛЕНИЕ. Однородными $k$-формами порядка $m$ на пространстве петель $\Omega M$ многообразия $M$ мы будем называть кососимметрические формы вида

$$
\begin{aligned}
& \omega\left(\xi_{1}, \ldots, \xi_{k}\right)=\int_{S^{1}}\left(\sum_{1} M_{i_{1} \cdots i_{k}}^{\left[n_{1} \cdots n_{k}\right]}(u)\left(\xi_{1}^{i_{1}}\right)_{\left(n_{1}\right)} \cdots\left(\xi_{k}^{i_{k}}\right)_{\left(n_{k}\right)}\right. \\
& \left.\quad+\sum_{2} M_{i_{1} \cdots i_{k} j_{1} \cdots j_{s}}^{\left[n_{1} \cdots n_{k} p_{1} \cdots p_{s}\right]}(u) u_{\left(p_{1}\right)}^{j_{1}} \cdots u_{\left(p_{s}\right)}^{j_{s}}\left(\xi_{1}^{i_{1}}\right)_{\left(n_{1}\right)} \cdots\left(\xi_{k}^{i_{k}}\right)_{\left(n_{k}\right)}\right) d x
\end{aligned}
$$

где первое суммирование проводится по всем $n_{1}, \ldots, n_{k}$, таким, что $n_{1}+\cdots$ $+n_{k}=m, n_{r} \geqslant 0$, а второе - по всем $s>0$ и по всем $n_{1}, \ldots, n_{k}, p_{1}, \ldots, p_{s}$, таким, что $n_{1}+\cdots+n_{k}+p_{1}+\cdots+p_{s}=m, n_{r} \geqslant 0, p_{l}>0$, и, кроме того, по всем $1 \leqslant i_{r} \leqslant N, 1 \leqslant r \leqslant k, 1 \leqslant j_{l} \leqslant N, 1 \leqslant l \leqslant s$. Здесь $M_{i_{1} \cdots i_{k} j_{1} \cdots j_{s}}^{\left[n_{1} \cdots p_{k} p_{1} \cdots p_{s}\right]}(u)$ и $M_{i_{1} \cdots i_{k}}^{\left[n_{1} \cdots n_{k}\right]}(u)$ - гладкие функции на многообразии $M$, а $\xi_{r}(x) \in T_{\gamma} \Omega M-$ векторные поля вдоль петли $\gamma(x)=\left\{u^{i}(x)\right\}$.

Мы используем здесь стандартные обозначения для полных производных порядка $n$ по $x: f_{(n)}=d^{n} f / d x^{n}$. Всегда можно считать, что $0<p_{1} \leqslant \cdots \leqslant p_{s}$ и, кроме того, $n_{1}=0$ (при $k>0$ ), так как в нашем случае $\int_{S^{1}}(d G / d x) d x=0$ для всех рассматриваемых функций $G\left(x, u(x), u_{x}(x), \ldots\right)$, заданных на пет$\operatorname{\pi яx} \gamma$.

Очевидно, что множество $\Omega_{[m]}^{k}$ всех однородных $k$-форм порлдка $m$ на пространстве петель $\Omega M$ является линейным пространством над $\mathbb{R}$, причем определение пространства $\Omega_{[m]}^{k}$ не зависит от локальных координат $\left\{u^{i}\right\}$, так как локальные замены координат $u^{i}=u^{i}\left(v^{1}, \ldots, v^{N}\right)$ на многообразии $M$ не выводят однородные $k$-формы порядка $m$ из этого класса. Число $m$ является инвариантом однородной формы относительно локальных замен координат на многообразии.

Мы будем использовать также следующие обозначения: $\Omega_{[m]}$ - линейное пространство всех однородных форм порлдка $m$ на пространстве петель $\Omega M$ многообразия $M, \Omega^{k}$ - линейное пространство всех однородных $k$-форм на $\Omega M, \Omega$ - линейное пространство всех однородных форм на $\Omega M$ :

$$
\Omega=\sum_{m \geqslant 0} \Omega_{[m]}=\sum_{k \geqslant 0} \Omega^{k}=\sum_{m \geqslant 0} \sum_{k \geqslant 0} \Omega_{[m]}^{k} .
$$




\section{§2. Комплексы однородных форм на пространствах петель гладких многообразий}

Дифференциал $d$ на однородных $k$-формах определим следующей формулой:

$$
\begin{aligned}
& (d \omega)\left(\xi_{1}, \ldots, \xi_{k+1}\right) \\
& =\sum_{q=1}^{k+1}(-1)^{q+1} \int_{S^{1}}\left(\xi_{q}^{i}\right)_{(r)}\left(\xi_{1}^{i_{1}}\right)_{\left(n_{1}\right)} \cdots\left(\xi_{q-1}^{i_{q-1}}\right)_{\left(n_{q-1}\right)}\left(\xi_{q+1}^{i_{q}}\right)_{\left(n_{q}\right)} \cdots\left(\xi_{k+1}^{i_{k}}\right)_{\left(n_{k}\right)} \\
& \times \frac{\partial\left(M_{i_{1} \cdots i_{k}}^{\left[n_{1} \cdots n_{k}\right]}(u)+M_{i_{1} \cdots i_{k} j_{1} \cdots j_{s}}^{\left[n_{1} \cdots n_{k} p_{1} \cdots p_{s}\right]}(u) u_{\left(p_{1}\right)}^{j_{1}} \cdots u_{\left(p_{s}\right)}^{j_{s}}\right)}{\partial u_{(r)}^{i}} d x
\end{aligned}
$$

ПРЕДложЕнИЕ. ДифФеренииал $d$ является линейным отображением на линейном пространстве $\Omega$ однородных форм и переводит однородную $k$-форму порядка $m$ в однородную $(k+1)$-форму порядка $m$ :

$$
d: \Omega_{[m]}^{k} \rightarrow \Omega_{[m]}^{k+1},
$$

причем $d^{2}=0$.

Таким образом, определен комплекс $(\Omega, d)$ однородных форм на пространстве петель $\Omega M$ :

$$
0 \stackrel{d}{\rightarrow} \Omega^{0} \stackrel{d}{\rightarrow} \Omega^{1} \stackrel{d}{\longrightarrow} \Omega^{2} \stackrel{d}{\rightarrow} \cdots
$$

Более того, для любого $m$ определен комплекс $\left(\Omega_{[m]}, d\right)$ однородных форм порядка $m$ на пространстве петель $\Omega M$ :

$$
0 \stackrel{d}{\longrightarrow} \Omega_{[m]}^{0} \stackrel{d}{\longrightarrow} \Omega_{[m]}^{1} \stackrel{d}{\longrightarrow} \Omega_{[m]}^{2} \stackrel{d}{\longrightarrow} \cdots .
$$

Введем обозначение $Z_{[m]}^{k}(\Omega M)$ для замкнутых $(d \omega=0)$ и соответственно $B_{[m]}^{k}(\Omega M)$ для точных $(\omega=d \alpha)$ однородных $k$-фрорм $\omega$ порядка $m$ на $\Omega M$ и определим группы когомологий нашего комплекса (2.3):

$$
H_{[m]}^{k}(\Omega M, \mathbb{R})=Z_{[m]}^{k}(\Omega M) / B_{[m]}^{k}(\Omega M) .
$$

ОПРЕДЕЛЕниЕ. Группы $H_{[m]}^{k}(\Omega M, \mathbb{R})$ будем называть группами однородньх когомологий порядка $m$ пространства петель $\Omega M$ гладкого многообразия $M$.

Введем также обозначение $Z^{k}(\Omega M)$ для всех замкнутых и соответственно $B^{k}(\Omega M)$ для всех точных однородных $k$-форм на $\Omega M$ и определим груnnь однородньх когомологий пространства петель $\Omega M$ гладкого многообразия $M$ формулой

$$
H^{k}(\Omega M, \mathbb{R})=Z^{k}(\Omega M) / B^{k}(\Omega M)
$$




\section{§3. Группы когомологий комплексов однородных форм на пространствах петель гладких многообразий}

Однородные когомологии нулевого порядка пространства петель $\Omega M$ совпадают с когомологиями де Рама многообразия $M$ :

$$
H_{[0]}^{i}(\Omega M, \mathbb{R})=H^{i}(M, \mathbb{R}) .
$$

Действительно, однородные $k$-формы нулевого порядка имеют вид

$$
\omega\left(\xi_{1}, \ldots, \xi_{k}\right)=\int_{S^{1}} M_{i_{1} \cdots i_{k}}(u) \xi_{1}^{i_{1}} \cdots \xi_{k}^{i_{k}} d x,
$$

где $M_{i_{1} \cdots i_{k}}(u)$ - произвольные гладкие кососимметрические ковариантные тензорные поля на многообразии $M$, а дифференциал $d$ (формула (2.1)) в этом случае действует на них как классический дифференциал де Рама:

$$
\begin{aligned}
(d \omega)\left(\xi_{1}, \ldots, \xi_{k+1}\right) & =\sum_{q=1}^{k+1}(-1)^{q+1} \int_{S^{1}} \xi_{q}^{i_{q}} \xi_{1}^{i_{1}} \cdots \xi_{q-1}^{i_{q-1}} \xi_{q+1}^{i_{q}} \cdots \xi_{k+1}^{i_{k}} \frac{\partial M_{i_{1} \cdots i_{k}}(u)}{\partial u^{i}} d x \\
& =\int_{S^{1}}(d M)_{i_{1} \cdots i_{k+1}}(u) \xi_{1}^{i_{1}} \cdots \xi_{k+1}^{i_{k+1}} d x \\
(d M)_{i_{1} \cdots i_{k+1}}(u) & =\sum_{q=1}^{k+1}(-1)^{q+1} \frac{\partial M_{i_{1} \cdots \hat{i}_{q} \cdots i_{k+1}}}{\partial u^{i_{q}}} .
\end{aligned}
$$

Таким образом, комплекс (2.2) является естественным обобщением классического комплекса де Рама на пространства петель гладких многообразий.

По-видимому, все группы когомологий комплекса однородных форм (2.2) должны выражаться через группы когомологий де Рама многообразия $M$. Мы рассмотрим здесь подробно вычисление первых групп когомологий при $m=1$. В частности, в этом случае имеет место следующая теорема о связи групп однородных когомологий первого порядка с группами когомологий де Рама.

TeOpema 1. Прu $i=0,1,2$

$$
H_{[1]}^{i}(\Omega M, \mathbb{R})=H^{i+1}(M, \mathbb{R}) .
$$

ДокаЗАТЕЛЬСтво. Рассмотрим однородные $k$-формы первого порядка на $\Omega M$

$$
\begin{aligned}
\omega\left(\xi_{1}, \ldots, \xi_{k}\right)=\int_{S^{1}}\left(\sum_{s=2}^{k} \xi_{1}^{i_{1}} M_{i_{1} \cdots i_{k}}^{[s]}(u)\right. & \xi_{2}^{i_{2}} \cdots\left(\xi_{s}^{i_{s}}\right)_{x} \cdots \xi_{k}^{i_{k}} \\
& \left.+\xi_{1}^{i_{1}} M_{i_{1} \cdots i_{k} j}(u) u_{x}^{j} \xi_{2}^{i_{2}} \cdots \xi_{k}^{i_{k}}\right) d x
\end{aligned}
$$

где $M_{i_{1} \cdots i_{k}}^{[s]}(u)$ и $M_{i_{1} \cdots i_{k} j}(u)$ - гладкие функции на многообразии $M$, удовлетворяющие соотношениям, обеспечивающим кососимметричность $k$-формы (3.3). 
Соответственно однородные 0-формы первого порядка - это функционалы $F$ на $\Omega M$ вида

$$
F=\int_{S^{1}} f_{i}(u) u_{x}^{i} d x
$$

где $f_{i}(u)$ - произвольное гладкое ковекторное поле на многообразии $M$. При этом отметим, что в рассматриваемом нами случае на пространстве петель интеграл вдоль петли от полной производной по $x$ всегда равен 0 :

$$
\int_{S^{1}}\left(\frac{d}{d x} f(u)\right) d x=\int_{S^{1}} \frac{\partial f}{\partial u^{i}} u_{x}^{i} d x=0,
$$

где $f(u)$ - произвольная функция на многообразии $M$.

Найдем условие замкнутости 0-формы первого порядка (3.4):

$$
(d F)(\xi) \equiv \int_{S^{1}} \xi^{i}\left(\frac{\partial f_{k}}{\partial u^{i}}-\frac{\partial f_{i}}{\partial u^{k}}\right) u_{x}^{k} d x,
$$

т. е. 0-форма (3.4) замкнута тогда и только тогда, когда выполнено соотношение

$$
\frac{\partial f_{k}}{\partial u^{i}}-\frac{\partial f_{i}}{\partial u^{k}}=0 .
$$

Точными в данном случае являются 0-формы, тождественно равные нулю, т.е., в силу (3.5), те 0-фрормы (3.4), для которых

$$
f_{i}=\frac{\partial f(u)}{\partial u^{i}}
$$

Соотношение (3.7) означает, что 1-форма $f_{i}(u) d u^{i}$ на многообразии $M$ является замкнутой, а соотношение (3.8) — что она является на нем точной 1-формой.

Таким образом, доказано, что $H_{[1]}^{0}(\Omega M, \mathbb{R})=H^{1}(M, \mathbb{R})$.

Однородные 1-формы первого порядка на пространстве петель имеют вид

$$
\omega(\xi)=\int_{S^{1}} \xi^{i} f_{i k}(u) u_{x}^{k} d x,
$$

где $f_{i k}(u)$ - произвольное гладкое ковариантное двухвалентное тензорное поле на многообразии $M$.

Найдем условия замкнутости однородных 1-форм первого порядка (3.9):

$$
\begin{aligned}
(d \omega)(\xi, \eta) & =\int_{S^{1}} \xi^{i}\left(\eta^{j} \frac{\partial f_{j k}}{\partial u^{i}} u_{x}^{k}-\left(\eta^{j} f_{j i}\right)_{x}\right) d x-\int_{S^{1}} \eta^{j}\left(\xi^{i} \frac{\partial f_{i k}}{\partial u^{j}} u_{x}^{k}-\left(\xi^{i} f_{i j}\right)_{x}\right) d x \\
& =\int_{S^{1}} \xi^{i}\left[\eta^{j}\left(\frac{\partial f_{j k}}{\partial u^{i}}-\frac{\partial f_{j i}}{\partial u^{k}}-\frac{\partial f_{i k}}{\partial u^{j}}\right) u_{x}^{k}-\eta_{x}^{j}\left(f_{j i}+f_{i j}\right)\right] d x,
\end{aligned}
$$

т. е. однородная 1-форма первого порядка (3.9) замкнута тогда и только тогда, когда выполнены соотношения

$$
\begin{gathered}
f_{j i}+f_{i j}=0 \\
\frac{\partial f_{j k}}{\partial u^{i}}-\frac{\partial f_{j i}}{\partial u^{k}}-\frac{\partial f_{i k}}{\partial u^{j}}=0 .
\end{gathered}
$$


Соотношения (3.11) и (3.12) в точности означают, что $f_{i j} d u^{i} \wedge d u^{j}$ замкнутая 2-форма на многообразии $M$. Из формулы (3.6) получаем, что однородная 1-форма (3.9) является точной тогда и только тогда, когда

$$
f_{i k}=\frac{\partial f_{k}}{\partial u^{i}}-\frac{\partial f_{i}}{\partial u^{k}}
$$

т.е. при условии, что $f_{i j} d u^{i} \wedge d u^{j}$ - точная 2-форма на многообразии $M$.

Таким образом, $H_{[1]}^{1}(\Omega M, \mathbb{R})=H^{2}(M, \mathbb{R})$.

Однородные 2-формы первого порядка имеют вид

$$
\omega(\xi, \eta)=\int_{S^{1}} \xi^{i}\left(g_{i j}(u)\left(\eta^{j}\right)_{x}+b_{i j k}(u) u_{x}^{k} \eta^{j}\right) d x,
$$

где $g_{i j}(u)$ — гладкое ковариантное двухвалентное тензорное поле на многообразии $M$, а $b_{i j k}(u)$ - гладкое поле на многообразии $M$, компоненты которого меняются по следующему закону при локальных заменах координат $u^{i}=u^{i}\left(v^{1}, \ldots, v^{N}\right)$ на многообразии:

$$
\tilde{b}_{p r s}(v)=b_{i j k}(u(v)) \frac{\partial u^{i}}{\partial v^{p}} \frac{\partial u^{j}}{\partial v^{r}} \frac{\partial u^{k}}{\partial v^{s}}+g_{i j}(u(v)) \frac{\partial u^{i}}{\partial v^{p}} \frac{\partial^{2} u^{j}}{\partial v^{r} \partial v^{s}} .
$$

В частности, если $g_{i j}(u)$ - невырожденная симметричная метрика на многообразии $M$ (т. е. если $\left(M, g_{i j}\right)$ - псевдориманово многообразие), то коэффициенты $b_{i j k}(u)$ определяют аффинную связность $\Gamma_{j k}^{i}(u)$ на многообразии $M$ :

$$
b_{i j k}(u)=g_{i s}(u) \Gamma_{j k}^{s}(u) .
$$

При этом для 2-формы (3.14) должны быть выполнены условия кососимметричности $\omega(\xi, \eta)=-\omega(\eta, \xi)$ :

$$
\begin{aligned}
\omega(\xi, \eta)+\omega(\eta, \xi) & =\int_{S^{1}}\left[\xi^{i} g_{i j}\left(\eta^{j}\right)_{x}+\eta^{j} g_{j i}\left(\xi^{i}\right)_{x}+\xi^{i}\left(b_{i j k}+b_{j i k}\right) u_{x}^{k} \eta^{j}\right] d x \\
& =\int_{S^{1}} \xi^{i}\left[\left(g_{i j}-g_{j i}\right)\left(\eta^{j}\right)_{x}+\left(b_{i j k}+b_{j i k}-\frac{\partial g_{j i}}{\partial u^{k}}\right) u_{x}^{k} \eta^{j}\right] d x=0,
\end{aligned}
$$

T.e.

$$
\begin{aligned}
g_{i j} & =g_{j i}, \\
\frac{\partial g_{i j}}{\partial u^{k}} & =b_{i j k}+b_{j i k} .
\end{aligned}
$$

Отметим, что в наиболее важном для приложений случае, когда $g_{i j}(u)-$ псевдориманова метрика на многообразии, соотношение (3.18) означает в точности, что связность, определяемая формулой (3.16), согласована с метрикой $g_{i j}(u)$ :

$$
\nabla_{k} g_{i j} \equiv \frac{\partial g_{i j}}{\partial u^{k}}-g_{i s} \Gamma_{j k}^{s}-g_{s j} \Gamma_{i k}^{s}=0
$$


Найдем условия замкнутости 2-формы (3.14):

$$
\begin{aligned}
(d \omega)(\xi, \eta, \zeta)=\int_{S^{1}}\left[\xi^{i}\right. & \left(\frac{\partial g_{j k}}{\partial u^{i}} \eta^{j}\left(\zeta^{k}\right)_{x}+\frac{\partial b_{j k s}}{\partial u^{i}} u_{x}^{s} \eta^{j} \zeta^{k}\right)+\left(\xi^{i}\right)_{x} b_{j k i} \eta^{j} \zeta^{k} \\
& -\eta^{j}\left(\frac{\partial g_{i k}}{\partial u^{j}} \xi^{i}\left(\zeta^{k}\right)_{x}+\frac{\partial b_{i k s}}{\partial u^{j}} u_{x}^{s} \xi^{i} \zeta^{k}\right)-\left(\eta^{j}\right)_{x} b_{i k j} \xi^{i} \zeta^{k} \\
& \left.+\zeta^{k}\left(\frac{\partial g_{i j}}{\partial u^{k}} \xi^{i}\left(\eta^{j}\right)_{x}+\frac{\partial b_{i j s}}{\partial u^{k}} u_{x}^{s} \xi^{i} \eta^{j}\right)+\left(\zeta^{k}\right)_{x} b_{i j k} \xi^{i} \eta^{j}\right] d x \\
=\int_{S^{1}} \xi^{i}[ & \left(\frac{\partial g_{i j}}{\partial u^{k}}-b_{j k i}-b_{i k j}\right)\left(\eta^{j}\right)_{x} \zeta^{k} \\
& +\left(\frac{\partial g_{j k}}{\partial u^{i}}-b_{j k i}-\frac{\partial g_{i k}}{\partial u^{j}}+b_{i j k}\right) \eta^{j}\left(\zeta^{k}\right)_{x} \\
& \left.+\left(\frac{\partial b_{j k s}}{\partial u^{i}}-\frac{\partial b_{j k i}}{\partial u^{s}}+\frac{\partial b_{i j s}}{\partial u^{k}}-\frac{\partial b_{i k s}}{\partial u^{j}}\right) u_{x}^{s} \eta^{j} \zeta^{k}\right] d x \equiv 0 .
\end{aligned}
$$

Таким образом, условия замкнутости 2-формы (3.14) при выполнении условий кососимметричности $(3.17),(3.18)$ эквивалентны соотношениям

$$
\begin{gathered}
\frac{\partial g_{i j}}{\partial u^{k}}=b_{i k j}+b_{j k i} \\
\frac{\partial b_{j k s}}{\partial u^{i}}-\frac{\partial b_{j k i}}{\partial u^{s}}+\frac{\partial b_{i j s}}{\partial u^{k}}-\frac{\partial b_{i k s}}{\partial u^{j}}=0 .
\end{gathered}
$$

Выясним дифференциально-геометрический смысл соотношений замкнутости (3.19) и (3.20) для кососимметрических 2-форм вида (3.14) (т.е. при выполнении условий (3.17) и (3.18)).

ТЕорема 2. Однородная 2-форма первого порядка (3.14) замкнута тогда u только тогда, когда $g_{i j}=g_{j i} u$

$$
b_{i j k}=\frac{1}{2}\left(\frac{\partial g_{i k}}{\partial u^{j}}+\frac{\partial g_{j i}}{\partial u^{k}}-\frac{\partial g_{j k}}{\partial u^{i}}+T_{i j k}(u)\right),
$$

где $T_{i j k}(u)$ - кососимметрический тензор, определяемьий произвольной замкнутой 3-формой $\alpha=T_{i j k}(u) d u^{i} \wedge d u^{j} \wedge d u^{k}$ на многообразии М. При этом 2-форма (3.14) является точной тогда и только тогда, когда $\alpha-$ произвольная точная 3-форма на многообразии $M$, т.е. $T_{i j k}(u)$ - тензорное поле, определяемое произвольной 2-формой $\beta=\beta_{i j}(u) d u^{i} \wedge d u^{j}$ на многообразии: $T_{i j k}=(d \beta)_{i j k}$.

ДокАЗАТЕльСтво. Введем $T_{i j k}(u)$ формулой

$$
T_{i j k}=b_{i j k}-b_{i k j} \text {. }
$$

Из закона преобразования коэффициентов $b_{i j k}(u)$ на многообразии $M(3.15)$ следует, что $T_{i j k}(u)$ - тензор на $M$. В том случае, когда $g_{i j}(u)$ - невырожденная метрика, $T_{i j k}(u)$ - тензор кручения (с нижними индексами) связности $\Gamma_{j k}^{s}(u)$, определяемой формулой $(3.16)$ :

$$
T_{i j k}=g_{i s} T_{j k}^{s}=g_{i s}\left(\Gamma_{j k}^{s}-\Gamma_{k j}^{s}\right) .
$$


Сравнивая формулы (3.18) и (3.19), получаем

$$
T_{i j k}=T_{j k i},
$$

т. е., поскольку в силу своего определения (3.22) тензор $T_{i j k}(u)$ всегда кососимметричен по паре последних индексов, соотношение (3.19) эквивалентно условию, что он кососимметричен по любой паре индексов и, следовательно, определяет 3-форму $\alpha=T_{i j k}(u) d u^{i} \wedge d u^{j} \wedge d u^{k}$ на многообразии $M$.

Докажем, что соотношение (3.20) эквивалентно условию, что 3-форма $\alpha$ на многообразии, определяемая кососимметрическим тензором $T_{i j k}(u)$, является замкнутой.

Введем новое обозначение:

$$
S_{i j s k}(u)=\frac{\partial b_{i j s}}{\partial u^{k}}-\frac{\partial b_{i j k}}{\partial u^{s}} .
$$

В силу определения функция $S_{i j s k}(u)$ кососимметрична по последней паре индексов

$$
S_{i j s k}=-S_{i j k s} .
$$

Кроме того, используя формулу (3.18), получаем, что она кососимметрична и по первой паре индексов:

$$
S_{i j s k}=\frac{\partial b_{i j s}}{\partial u^{k}}-\frac{\partial b_{i j k}}{\partial u^{s}}=-\frac{\partial b_{j i s}}{\partial u^{k}}+\frac{\partial^{2} g_{i j}}{\partial u^{s} \partial u^{k}}+\frac{\partial b_{j i k}}{\partial u^{s}}-\frac{\partial^{2} g_{i j}}{\partial u^{s} \partial u^{k}}=-S_{j i s k} .
$$

Выведем ряд необходимых соотношений:

$$
\begin{aligned}
S_{i j k m}+S_{i k m j}+S_{i m j k} & =\frac{\partial b_{i j k}}{\partial u^{m}}-\frac{\partial b_{i j m}}{\partial u^{k}}+\frac{\partial b_{i k m}}{\partial u^{j}}-\frac{\partial b_{i k j}}{\partial u^{m}}+\frac{\partial b_{i m j}}{\partial u^{k}}-\frac{\partial b_{i m k}}{\partial u^{j}} \\
& =\frac{\partial T_{i j k}}{\partial u^{m}}-\frac{\partial T_{i j m}}{\partial u^{k}}+\frac{\partial T_{i k m}}{\partial u^{j}} .
\end{aligned}
$$

Отсюда получаем, используя указанные выше свойства (3.25) и (3.26) кососимметричности функции $S_{i j k m}(u)$ и кососимметричность тензора $T_{i j k}(u)$,

$$
\begin{aligned}
2 S_{i j k m}-2 S_{m k j i}= & S_{i j k m}+S_{i k m j}+S_{i m j k}+S_{i j k m}+S_{k j m i}+S_{j m k i} \\
& \quad-S_{j m k i}-S_{i m j k}-S_{m k j i}-S_{k j m i}-S_{i k m j}-S_{m k j i} \\
= & S_{i j k m}+S_{i k m j}+S_{i m j k}+S_{j i m k}+S_{j k i m}+S_{j m k i} \\
& \quad-S_{m j i k}-S_{m i k j}-S_{m k j i}-S_{k j m i}-S_{k i j m}-S_{k m i j} \\
= & \frac{\partial T_{i j k}}{\partial u^{m}}-\frac{\partial T_{i j m}}{\partial u^{k}}+\frac{\partial T_{i k m}}{\partial u^{j}}+\frac{\partial T_{j i m}}{\partial u^{k}}-\frac{\partial T_{j i k}}{\partial u^{m}}+\frac{\partial T_{j m k}}{\partial u^{i}} \\
& \quad-\frac{\partial T_{m j i}}{\partial u^{k}}+\frac{\partial T_{m j k}}{\partial u^{i}}-\frac{\partial T_{m i k}}{\partial u^{j}}-\frac{\partial T_{k j m}}{\partial u^{i}}+\frac{\partial T_{k j i}}{\partial u^{m}}-\frac{\partial T_{k m i}}{\partial u^{j}} \\
= & \frac{\partial T_{j i m}}{\partial u^{k}}+\frac{\partial T_{j k i}}{\partial u^{m}}-\frac{\partial T_{m k j}}{\partial u^{i}}-\frac{\partial T_{k m i}}{\partial u^{j}} .
\end{aligned}
$$

Соотношение (3.20) можно переписать в виде

$$
S_{j k s i}-S_{i s k j}+\frac{\partial T_{i j s}}{\partial u^{k}}-\frac{\partial T_{i k s}}{\partial u^{j}}=0 .
$$


Теперь с помощью формулы (3.28) преобразуем соотношение (3.20):

$$
\begin{aligned}
0 & =S_{i j k m}-S_{m k j i}+\frac{\partial T_{m i k}}{\partial u^{j}}-\frac{\partial T_{m j k}}{\partial u^{i}} \\
& =\frac{1}{2}\left(\frac{\partial T_{j i m}}{\partial u^{k}}+\frac{\partial T_{j k i}}{\partial u^{m}}-\frac{\partial T_{m k j}}{\partial u^{i}}-\frac{\partial T_{k m i}}{\partial u^{j}}\right)+\frac{\partial T_{m i k}}{\partial u^{j}}-\frac{\partial T_{m j k}}{\partial u^{i}} \\
& =\frac{1}{2}\left(\frac{\partial T_{j i m}}{\partial u^{k}}+\frac{\partial T_{j k i}}{\partial u^{m}}+\frac{\partial T_{m k j}}{\partial u^{i}}+\frac{\partial T_{k m i}}{\partial u^{j}}\right),
\end{aligned}
$$

т.е. соотношение (3.20) эквивалентно условию замкнутости 3-формы $\alpha$, определяемой кососимметрическим тензором $T_{i j k}(u)$ на многообразии $M$ :

$$
(d T)_{i j k m} \equiv \frac{\partial T_{j k m}}{\partial u^{i}}-\frac{\partial T_{i k m}}{\partial u^{j}}+\frac{\partial T_{i j m}}{\partial u^{k}}-\frac{\partial T_{i j k}}{\partial u^{m}}=0
$$

или $d \alpha=0$.

Переставляя индексы в (3.18), получаем

$$
\begin{aligned}
& \frac{\partial g_{j k}}{\partial u^{i}}=b_{j k i}+b_{k j i}, \\
& \frac{\partial g_{k i}}{\partial u^{j}}=b_{k i j}+b_{i k j} .
\end{aligned}
$$

Рассматривая теперь линейную комбинацию $(3.33)+(3.32)-(3.18)$ этих формул, получаем

$$
2 b_{k i j}+T_{k j i}+T_{j k i}+T_{i k j}=-\frac{\partial g_{i j}}{\partial u^{k}}+\frac{\partial g_{j k}}{\partial u^{i}}+\frac{\partial g_{k i}}{\partial u^{j}},
$$

T. e.

$$
b_{k i j}=\frac{1}{2}\left(\frac{\partial g_{k j}}{\partial u^{i}}+\frac{\partial g_{i k}}{\partial u^{j}}-\frac{\partial g_{i j}}{\partial u^{k}}+T_{k i j}\right) .
$$

Пусть теперь $g_{i j}(u)$ - произвольный симметрический тензор, $T_{i j k}(u)$ кососимметрический тензор, определяемый произвольной замкнутой 3-формой $\alpha=T_{i j k}(u) d u^{i} \wedge d u^{j} \wedge d u^{k}$ на многообразии $M$, а коэффициенты $b_{i j k}(u)$ определены формулой (3.34). Можно проверить, что соотношения (3.18), (3.19), (3.20), (3.22) выполняются.

Из формулы (3.10) следует, что однородная 2-форма (3.14) является точной тогда и только тогда, когда

$$
\begin{aligned}
g_{i j} & =f_{i j}+f_{j i}, \\
b_{i j k} & =\frac{\partial f_{j i}}{\partial u^{k}}+\frac{\partial f_{i k}}{\partial u^{j}}-\frac{\partial f_{j k}}{\partial u^{i}},
\end{aligned}
$$

где $f_{i j}(u)$ - произвольное гладкое тензорное поле на многообразии $M$.

Полагая $\beta_{i j}=f_{j i}-f_{i j}$, получаем, что для точной 2-формы (3.14)

$$
b_{i j k}=\frac{1}{2}\left(\frac{\partial g_{j i}}{\partial u^{k}}+\frac{\partial g_{i k}}{\partial u^{j}}-\frac{\partial g_{j k}}{\partial u^{i}}-\frac{\partial \beta_{j i}}{\partial u^{k}}-\frac{\partial \beta_{i k}}{\partial u^{j}}+\frac{\partial \beta_{j k}}{\partial u^{i}}\right),
$$


где $g_{i j}(u)$ - произвольное гладкое симметричное тензорное поле, а $\beta_{i j}(u)$ - произвольное гладкое кососимметрическое тензорное поле на многообразии $M$.

Таким образом, $H_{[1]}^{2}(\Omega M, \mathbb{R})=H^{3}(M, \mathbb{R})$. Теоремы 2 и 1 доказаны.

Отметим, что однородные $k$-формы первого порядка инвариантны относительно действия группы $\operatorname{Diff}^{+}\left(S^{1}\right)$ диффеоморфизмов окружности $S^{1}$, сохраняющих ориентацию, что важно с точки зрения приложений в теории замкнутых бозонных струн в искривленном $N$-мерном пространстве-времени $M$ с метрикой (гравитационным полем) $g_{i j}$, конфигурационным пространством которых является пространство петель $\Omega M$ псевдориманова многообразия $\left(M, g_{i j}\right)$ (требование инвариантности или независимости от параметризации петли является необходимым в теории бозонных струн для физически осмысленных объектов на конфигурационном пространстве $\Omega M)$.

Однородные симплектические структуры, изучавшиеся автором в [19-24], отвечают в точности замкнутым однородным 2-формам на $\Omega M$, т. е. являются элементами $Z_{[m]}^{2}(\Omega M)$.

Развитый в данной статье подход позволяет вычислить явно (выразить через когомологии де Рама многообразия) и высшие группы $(i>2)$ однородных когомологий $H_{[m]}^{i}(\Omega M, \mathbb{R})$ пространств петель, в том числе старших порядков $m \geqslant 2$, хотя это и требует большого объема вычислений.

Отметим, что сдвиг на $m=1$ в группах когомологий в формуле $(3.2)$, вообще говоря, не является общим фактом для любых $i$ и $m$. По крайней мере, несложно показать, что

$$
H_{[2]}^{0}(\Omega M, \mathbb{R})=0 .
$$

Действительно, однородные 0-формы второго порядка - это функционалы вида

$$
F=\int_{S^{1}} b_{i j}(u) u_{x}^{i} u_{x}^{j} d x,
$$

где $b_{i j}(u)$ - произвольное гладкое симметричное тензорное поле на $M$.

Найдем условия замкнутости 0-формы (3.38):

$$
\begin{aligned}
(d F)(\xi) & =\int_{S^{1}} \xi^{i}\left(\frac{\partial b_{k j}}{\partial u^{i}} u_{x}^{k} u_{x}^{j}-\left(b_{i j} u_{x}^{j}\right)_{x}-\left(b_{j i} u_{x}^{j}\right)_{x}\right) d x \\
& =\int_{S^{1}} \xi^{i}\left[\left(-b_{i j}-b_{j i}\right) u_{x x}^{j}+\left(\frac{\partial b_{k j}}{\partial u^{i}}-\frac{\partial b_{i j}}{\partial u^{k}}-\frac{\partial b_{j i}}{\partial u^{k}}\right) u_{x}^{k} u_{x}^{j}\right] d x \equiv 0 .
\end{aligned}
$$

Таким образом, для любой замкнутой однородной 0-формы второго порядка (3.38) должно выполняться соотношение $b_{i j}=0$, т. е. она обязательно является тождественно нулевой.

\section{ЛИТЕРАТУРА}

1. Мохов О. И. О комплексах однородных форм на пространствах петель гладких многообразий и их группах когомологий. УМН, 51, вып. 2, 141-142 (1996). 
2. Дубровин Б. А., Новиков С. П. Гамильтонов формализм одномерных систем гидродинамического типа и метод усреднения Боголюбова-Уизема. ДАН CCCP, 270, № 4, 781-785 (1983).

3. Дубровин Б. А., Новиков С. П. Гидродинамика слабо деформированных солитонных решеток. Дифференциальная геометрия и гамильтонова теория. УМН, 44, вып. 6, 29-98 (1989).

4. Dubrovin B. A., Novikov S. P. Hydrodynamics of soliton lattices. Sov. Sci. Rev.. Sec. C, Math. Phys., Vol. 9, Part 4, 1993, pp. 1-136.

5. Царев С. П. Геометрия гамильтоновых систем гидродинамического типа. Обобщенный метод годографа. Изв. АН СССР, сер. матем., 54, вып. 5, 1048-1068 (1990).

6. Maltsev A. Ya. The conservation of the Hamiltonian structures in Whitham's method of averaging. Preprint, solv-int/9611008.

7. Мальиев $A$. $Я$. Усреднение локальных теоретико-полевых скобок Пуассона. УМН, 52, вып. 2, 177-178 (1997).

8. Мохов О.И., Ферапонтов Е. В. О нелокальных гамильтоновых операторах гидродинамического типа, связанных с метриками постоянной кривизны. УМН, 45, вып. 3, 191-192 (1990).

9. Mokhov O. I. Hamiltonian systems of hydrodynamic type and constant curvature metrics. Phys. Lett. A, 166, No. 3, 4, 215-216 (1992).

10. Ферапонтов E. B. Дифференциальная геометрия нелокальных гамильтоновых операторов гидродинамического типа. Функц. анализ и его прил., 25, вып. 3, 37-49 (1991).

11. Мохов О. И., Ферапонтов Е. В. Гамильтоновы пары, порождаемые кососимметричными тензорами Киллинга на пространствах постоянной кривизны. Функц. анализ и его прил., 28, вып. 2, 60-63 (1994).

12. Дубровин Б. А., Новиков С. П. О скобках Пуассона гидродинамического типа. ДАН СССР, 279, № 2, 294-297 (1984).

13. Потемин Г. В. О скобках Пуассона дифференциально-геометрического типа. ДАН СССР, 286, № 1, 39-42 (1986).

14. Потемин Г. В. Некоторые вопросы дифференциальной геометрии и алгебраической геометрии в теории солитонов. Дисс. к.ф.-м.н., МГУ, М., 1991.

15. Doyle $P$. $W$. Differential geometric Poisson bivectors in one space variable. J. Math. Phys., 34, No. 4, 1314-1338 (1993).

16. Mokhov O. I., Nutku Y. Homogeneous Poisson brackets of Dubrovin-Novikov type and their nonlocal generalizations. Preprint, TÜBITTAK - Marmara Research Center, Research Institute for Basic Sciences, Gebze, Turkey, 1996.

17. Ferapontov E. V., Galvão C. A. P., Mokhov O. I., Nutku Y. Bi-Hamiltonian structure of equations of associativity in 2-d topological field theory. Commun. Math. Phys., 186, 649-669 (1997).

18. Мохов О. И. О скобках Пуассона типа Дубровина-Новикова (ДН-скобки). Функц. анализ и его прил., 22, вып. 4, 92-93 (1988).

19. Мохов О. И. Симплектические формы на пространстве петель и риманова геометрия. Функц. анализ и его прил., 24, вып. 3, 86-87 (1990).

20. Мохов О. И. Однородные симплектические структуры второго порядка на пространствах петель и симплектические связности. Функц. анализ и его прил., 25, вып. 2, 65-67 (1991). 
21. Mokhov O. I. Symplectic and Poisson geometry on loop spaces of manifolds and nonlinear equations. In: Topics in topology and mathematical physics (S. P. Novikov ed.), Amer. Math. Soc. Transl., ser. 2, Vol. 170, Providence, RI, 1995, pp. 121-151; hep-th/9503076.

22. Мохов О. И. Симплектические и пуассоновы структуры на пространствах петель гладких многообразий и интегрируемые системы. Дисс. д.ф.-м.н., МИАН, M., 1996.

23. Mokhov O. I. Two-dimensional nonlinear sigma-models and symplectic geometry on loop spaces of (pseudo)-Riemannian manifolds. In: Nonlinear Evolution Equations and Dynamical Systems, Proc. 8th Internat. Workshop on Nonlinear Evolution Equations and Dynamical Systems (NEEDS'92), July 6-17, 1992, Dubna, V. Makhankov, I. Puzynin, and O. Pashaev eds., World Scientific Publishing, Singapore, 1993, pp. 444-456; hep-th/9301048.

24. Mokhov O. I. Poisson and symplectic geometry on loop spaces of smooth manifolds. In: Geometry from the Pacific Rim, Proc. Pacific Rim Geometry Conference ... National University of Singapore, Republic of Singapore, December 12-17, 1994, A. J. Berrick, B. Loo, H.-Y. Wang eds., Walter de Gruyter \& Co., Berlin, 1997, pp. $285-309$.

Центр нелинейных исследований при Институте теоретической физики им. Л. Д. Ландау РАН e-mail: mokhov@genesis.mi.ras.ru, mokhov@landau.ac.ru
Поступило в редакцию 28 января 1997 г. 